\title{
The Efficacy of Salinomycin Against Experimentally Infected Broiler Chickens with Field Isolates of Eimeria tenella in Khartoum State, Sudan
}

\author{
Mortada Mahgoub Osman Elhassan ${ }^{1}$, Mona Abdelrahman Mohamed Khaier, ", \\ Elgailani Ali Elamin ${ }^{2}$ \\ ${ }^{1}$ College of Veterinary Medicine, University of Bahri, Khartoum, Sudan \\ ${ }^{2}$ Faculty of Veterinary Medicine, University of Khartoum, Khartoum, Sudan
}

Email address:

munakhaier@gmail.com(M. A. M. Khaier)

${ }^{*}$ Corresponding author

\section{To cite this article:}

Mortada Mahgoub Osman Elhassan, Mona Abdelrahman Mohamed Khaier, Elgailani Ali Elamin. The Efficacy of Salinomycin Against Experimentally Infected Broiler Chickens with Field Isolates of Eimeria tenella in Khartoum State, Sudan. Animal and Veterinary Sciences. Vol. 8, No. 4, 2020, pp. 71-75. doi: 10.11648/j.avs.20200804.11

Received: June 13, 2020; Accepted: June 28, 2020; Published: July 13, 2020

\begin{abstract}
Administration of feed-based anticoccidials, as preventive measure, is one of the great successes in disease control for the ever-expanding poultry industry. The present study aimed at evaluating the efficacy of salinomycin medication against experimental infection with Eimeria tenella isolated from a local farm in Khartoum, Sudan. Forty-five one-day-old male broiler chicks (Ross 308) were randomized into 3 equal sets (control, infected, and medicated treatment groups). Infection was established by inoculating each of 36 birds with $1 \times 10^{5}$ sporulated oocysts when they were 24 days old. After 6 days, 9 birds of each group were bled for sera and sacrificed for post mortem examination. Birds of infected non-medicated group developed caecal lesions typical of E. tenella infection. Birds of salinomycin treatment at the concentration of $60 \mathrm{gm} / \mathrm{ton}$ of feed had highly diminished lesion scores (0.33) compared to infected non-medicated bird (3.00). Packed cell volume (PCV), triglycerides and cholesterol in birds medicated with salinomycin did not show marked difference as compared to broilers in negative control. There was no significant difference $(\mathrm{P}>0.05)$ in the levels of total protein and the concentration of calcium and phosphorus amongst experimental groups. It is concluded that salinomycin averted the negative impact of coccidiosis caused by E. tenella on broiler chicks; indicating that it could be recommended for establishing an efficient coccidian control programs under Sudan conditions.
\end{abstract}

Keywords: Broilers, Efficacy, Eimeria tenella, Salinomycin, Sudan

\section{Introduction}

Modern poultry industry has made big strides and become an important part of economic activity in many countries [1] However, the industry is exposed to high economic losses caused by coccidiosis, a disease of poultry worldwide induced by protozoan parasites of genus Eimeria [2] The global annual cost due to coccidiosis has been recently estimated to exceed 3 billion US\$ [3]. Costs are largely ascribed to decreased flock performance, prophylactic medication protocols, as well as the intercurrent infections such as necrotic enteritis $[4,5]$.

The most pathogenic species of coccidia which known to inhabit the lining of the ceca is Eimeria tenella. It is considered as the major cause of coccidiosis [6] and known to invade primarily growing chickens, causing considerable financial losses to poultry industry [7]. Studies conducted in Egypt and Sudan have shown that E. tenella was the most prevalent species in those countries $[8,9]$.

Administration of feed-based anticoccidials as preventives is one of the great successes in disease control for the everexpanding poultry industry [10]. Such anticoccidials can be broadly divided into two categories, synthetic agents produced by chemical synthesis that include compounds such as Dicluzuril, and ionophores that produced by fermentation such as salinomycin [11]. Nonetheless, prolonged use of 
these drugs has led to the emergence of drug resistant strains of Eimeria [12]. Despite the emerging resistance, adding anticoccidial drugs to poultry feeds remains essential to successful poultry production and is most likely to continue in the foreseeable future [13].

Salinomycin, a polyether ionophore, has been successfully used in Sudan as anticoccidial agent added to feed for more than two decades. However, very little is known on the resistance against such medicament developed by the existing coccidia. The objective of this study was to determine the efficacy of salinomycin against $E$. tenella isolated from local farm in Khartoum, Sudan.

\section{Materials and Methods}

\subsection{Field Isolation of the Parasite}

The oocysts utilized in this study were provided by Dr. Mona, College of Veterinary Medicine, University of Bahri. The oocysts represent a well-characterized 4 field isolates of E. tenella collected during the year 2017 in Khartoum State, Sudan. They were sequenced and deposited in the National Center of Biotechnology and Information (NCBI) with the accession numbers, MF497437, MF497438, MF497439 and MF497440. The number of oocysts per $1 \mathrm{ml}$ of the preservative solution (2.5\% potassium dichromate) was calculated using Mc Master counting technique.

\subsection{Propagation of Oocysts}

In order to obtain sufficient oocysts for experimental infection, five broiler chicks (aged 15 days) were inoculated with $1 \times 10^{5}$ oocysts. Ten days post infection, faeces were collected on daily basis till no oocyst seen. Sporulation and purification of the oocysts were done using standard techniques [14].

\subsection{Chicken Management}

The experiment was conducted in a closed-system house in which 54 one-day-old male broiler chicks (Ross 308) were reared. Birds were provided with starter diet, free of anticoccidial agents, until 21 days of age. Chicks were then given the experimental diet formulated as finisher feed from day 22 to 28 . Both starter and finisher rations were based on sorghum and groundnut cake, and formulated according to the guidelines of NRC [15].

Each pen was equipped with wood shavings in the floor, fountain drinker and tube feeder. Continuous lighting was provided throughout the starter period as well as the experiment duration. The temperature in the house at arrival was $33^{\circ} \mathrm{C}$ and then gradually adjusted to $26 \mathrm{C}^{\circ}$ during the first seven days of age.

\subsection{Experimental Design}

On day 22, chicks were randomly allocated into three groups (18 birds each) using a complete randomized design. Each group had three replicates (6 birds each). Each replicate was represented by a floor pen (dimensions $1 \times 0.75 \mathrm{~m})$. Birds in each group were initially weighed, and then given finisher diet either with or without salinomycin. The three treatments were: Group A, negative control, non-infected with coccidia and non-medicated; group B, positive control, infected with coccidia and non-medicated; group $\mathrm{C}$, infected with coccidia and medicated with salinomycin $\left(60 \mathrm{gm} /\right.$ ton feed; Sacox ${ }^{\circledR}$, Huvepharma, Bulgaria) as recommended by the manufacturer.

Before applying the coccidian infection, birds were allowed to acclimatize to experimental diet for two days (days 22-23). Each bird of infected groups was then inoculated orally with $1 \times 10^{5}$ sporulated oocyst of E. tenella in $1 \mathrm{ml}$ water using automatic pipette. The number of sporulated oocysts per $\mathrm{ml}$ was checked prior to inoculation using Mc Master counting technique.

Six days post inoculation, blood samples were collected from 3 birds randomly selected in each replicate. Therefore, each group was represented by 9 birds. The samples were then used for blood parameter tests including PCV, total proteins, triglycerides, cholesterol, calcium and phosphorus.

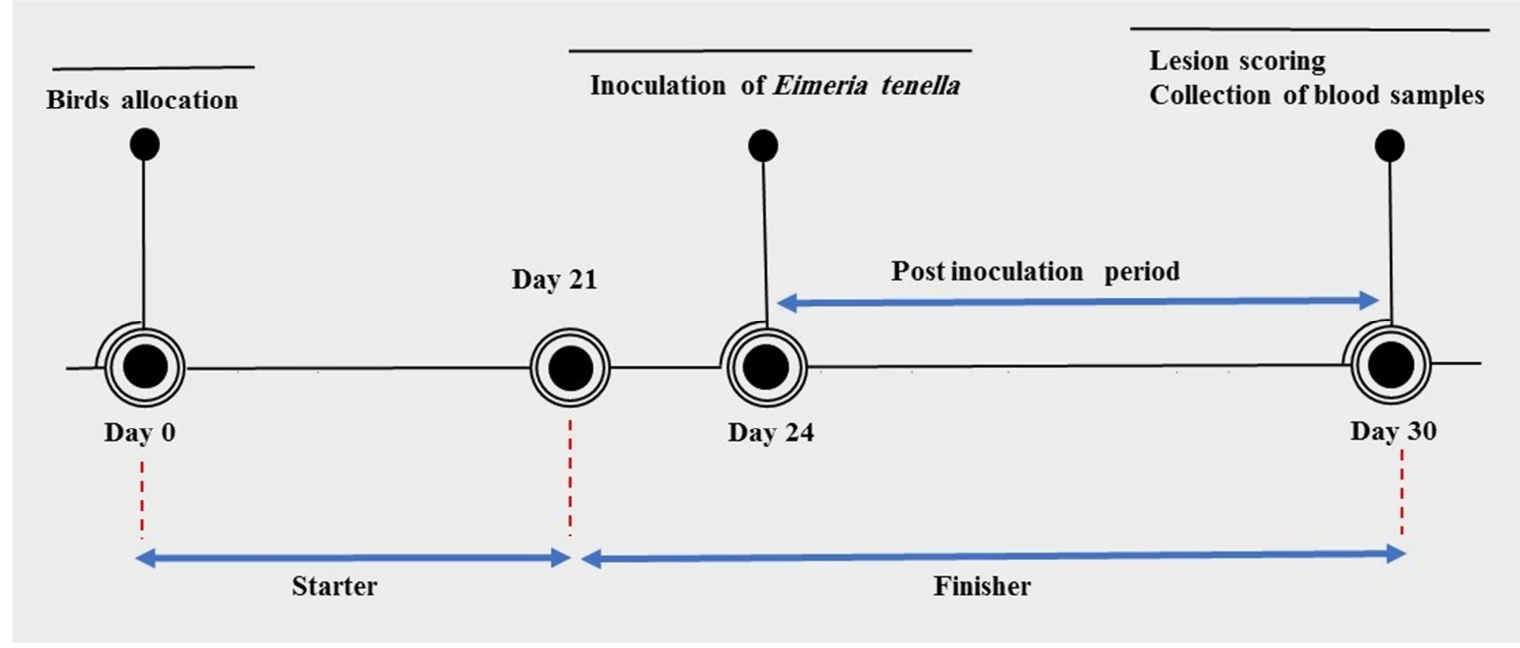

Figure 1. Time-line chart showing the procedures and methods applied throughout the rearing period of the broiler chicks (0-30 days). 
Afterwards, the same broilers were necropsied for caecal lesion score according to the method given by Johnson and Reid [16]. The sensitivity of E. tenella to salinomycin was established based on the formula given by Peek and Landman [17]. Briefly, the drug sensitivity was determined by the reduction of the mean lesion score of infected medicated group as compared to positive control group. To this end, the following formula was applied: 100\% - (mean lesion score of infected and medicated group/ mean lesion score of the positive control group $\times 100 \%$ ). Figure 1 displays a time-line chart showing the procedures and methods applied throughout the rearing period of the broiler chicks (0-30 days).

\subsection{Statistical Analysis}

Data were analyzed using Statistical Software Package (SPSS, version 21). Because the data of lesion scores were not distributed normally, they were analyzed by KruskalWallis nonparametric test. $p<0.01$ was considered statistically significant. Data of blood parameters were analyzed using one-way ANOVA. Significance of differences between the means was examined using Duncan's multiple range tests. $\mathrm{p}<0.05$ was considered statistically significant.

\section{Results}

As it can be seen in table 1, the infection caused characteristic lesions in chickens of the positive control with score 3 out of 4 . In addition, gross lesions were highly diminished $(\mathrm{p}<0.05)$ by infected-medicated treatment group (score 0.00) as compared with experimentally infectedunmedicated control. The sensitivity of E. tenella isolates to dietary addition of salinomycin was $89 \%$.

Table 2 shows the blood parameters of 29-day old broiler chicks in the different treatment groups. The infected unmedicated control group showed significant decrease in $\mathrm{PCV}$, triglycerides and cholesterol as compared to negative control $(\mathrm{p}<0.05)$, whereas PCV of infected medicated birds with salinomycin had no significant difference as compared to birds in the negative control group. The infected birds medicated with salinomycin revealed insignificant difference in the levels of triglycerides and cholesterol $(p>0.05)$ as compared with uninfected unmedicated control. Remarkable decrease in triglycerides and cholesterol $(\mathrm{p}<0.05)$ was observed in positive control group as compared with negative control one. There was no significant difference in the levels of total protein, calcium and phosphorus between the groups of the present experiment $(\mathrm{P}>0.05)$.

Table 1. The effect of salinomycin on gross caecal lesion scores and the sensitivity of Eimeria tenella to salinomycin ( $n=9)$.

\begin{tabular}{llll}
\hline Treatment & Lesion scores $^{\mathbf{1}}$ & Median lesion score & Mean lesion score $^{2}$ \\
\hline A & 000000000 & $0.00^{\mathrm{a}}$ & 0.00 \\
$\mathrm{~B}$ & 444333330 & $3.00^{\mathrm{b}}$ & 3.00 \\
$\mathrm{C}$ & 210000000 & $0.00^{\mathrm{a}}$ & 0.33 \\
\hline
\end{tabular}

A: Negative control, non-medicated and non-infected

B: Positive control, non-medicated and infected with coccidia

C: Medicated with Salinomycin and infected

${ }^{1}$ Nine birds from each group

${ }^{2}$ Standard error of mean $=0.27$

a , b, Medians with different superscript are significantly different $(\mathrm{P}<0.01)$

Table 2. Blood parameters of 29-day old broiler chicks in different treatment groups $(n=9)$.

\begin{tabular}{lllllll}
\hline Treatment & PCV\% & Total Protein mg/dl & Triglycerides mg/dl & Cholesterol mg/dL & Calcium mg/dl & Phosphorus mg/dl \\
\hline A & $31.3^{\mathrm{a}}$ & 1.84 & $77.5^{\mathrm{a}}$ & $125.5^{\mathrm{a}}$ & 7.00 & 2.36 \\
B & $26.9^{\mathrm{b}}$ & 1.64 & $44.9^{\mathrm{b}}$ & $86.8^{\mathrm{b}}$ & 5.89 & 2.03 \\
C & $28.6^{\mathrm{ab}}$ & 1.60 & $56.6^{\mathrm{ab}}$ & $107.6^{\mathrm{ab}}$ & 5.88 & 1.86 \\
\hline
\end{tabular}

A: Negative control, non-medicated and non-infected

B: Positive control, non-medicated and infected with coccidia

C: Medicated with Salinomycin and infected

PCV: Packed cell volume

a , b , c Means with different superscript are significantly different $(\mathrm{P}<0.05)$

\section{Discussion}

The present study revealed that it was possible to establish and produce typical caecal coccidiosis in experimentally infected chickens using a local isolate obtained from naturally infected chickens in Khartoum State, Sudan. Such infections have been successfully applied in several African countries to examine the pathogenicity and anticoccidial sensitivity of Eimeria species in chickens [18-20].

In this study, as shown by the reduction of gross lesion score, in-feed salinomycin reduced intestinal damage caused by $E$. tenella remarkably. In addition, the parasite displayed high sensitivity to the in-feed salinomycin. These findings are in line with previous studies conducted on broilers [21, 22].

Earlier studies demonstrated that the PCV was depressed by E. tenella infection $[23,24]$ whereas positive effect on PCV 
was observed when salinomycin administered to infected chicks [25]. This is in agreement with the present findings.

The species E. tenella has been reported to alleviate total serum protein of infected birds [23, 26]. This reduction plausibly as a consequence of gut damage which lead to reduced absorption of amino acids [27]. However, there was no discernable difference in the levels of total protein amongst the treatment groups in the present study.

In this study, unlike infected birds received salinomycin, low level of cholesterol was observed in infected unmedicated birds compared to uninfected unmedicated chicks. In accord with the present findings, earlier workers reported a decreased level of cholesterol when broilers infected with $E$. necatrix; they anticipated that the reduction of cholesterol caused by the parasite might possibly affects the synthesis of steroids, vitamin D and bile salts [28].

In agreement with the present study, significant decrease in the level of triglyceride has been reported during the infection with E. tenella [26]. Nevertheless, salinomycin in this study has successfully maintained the level of triglycerides after broilers being infected with E. tenella.

In the overall, it is well known that ionophores have been the first choice to control coccidiosis due to the slow development of resistance. This in addition to the fact that they do not completely suppress parasite growth and subsequently allowing the establishment of host immunity [3]. Although the anticoccidial salinomycin used in this study reduced the negative impact of coccidiosis caused by $E$. tenella, it is noteworthy that drug resistance still raises a great concern. This is because resistance to the available ionophores has been reported to become widespread [29]. Thus, it crucial to delay the emergence of drug resistance as much as possible. This could be primarily achieved by applying rotational programs that utilize different anticoccidial agents $[6,30]$. Another approach to overcome drug-resistance is the use of live oocysts vaccines that contain drug-sensitive strains of Eimeria spp; the ultimate goal of such application is to increase the number of drug sensitive strains in litter [31].

\section{Conclusions}

The local isolate of Eimeria tenella was shown to be of high morbidity and typical pathology. The anticoccidial salinomycin used in this clinical trial reduced the negative impact of coccidiosis caused by E. tenella on broiler chicks. The medicament exhibited a good potential to avert the caecal damage as well as maintaining the normal levels of serum protein, cholesterol, and triglyceride when broilers were infected with E. tenella. Therefore, salinomycin could be recommended for establishing an efficient coccidian control programs under Sudan conditions.

\section{Acknowledgements}

The authors gratefully acknowledge Doctors' Company LTD, Khartoum, Sudan for funding this research.

\section{References}

[1] Fesseha H (2019). Probiotics and its potential role in poultry production: a review. Vet Med - Open J.; 4 (2): 69-76.

[2] Firouzi S, Mosleh N, Tohidi Far SS, Taebipur MJ and Farjani Kish G (2014). Efficacy of anticoccidial vaccination of chickens via different routes: a comparative study. Bulg $J$ Vet Med.; 17 (4): 293-301.

[3] Noack S, Chapman HD and Selzer PM (2019). Anticoccidial drugs of the livestock industry. Parasitol Res.; 118 (7): 2009-2026.

[4] Price KR (2012). Use of live vaccines for coccidiosis control in replacement layer pullets. J Appl Poult Res.; 21 (3): 679-692.

[5] Williams RB (2005). Intercurrent coccidiosis and necrotic enteritis of chickens: Rational, integrated disease management by maintenance of gut integrity. Avian Pathol.; 34 (3): 159-180.

[6] Blake DP, Clark EL, Macdonald SE, Thenmozhi V, Kundu K, Garg R. (2015). Population, genetic, and antigenic diversity of the apicomplexan Eimeria tenella and their relevance to vaccine development. National Academy of Sciences. 112 (38): E5343-5350.

[7] Shirley MW, Smith AL and Blake DP (2007). Challenges in the successful control of the avian coccidia. Vaccine; 25: 5540-5547.

[8] Desouky A and Sultan K (2015). A preliminary survey on Eimeria species infecting chicken in Gharbia Governorate, Mid-Delta of Egypt. Egyptian Veterinary Medical Society of Parasitology Journa.; 11: 59-65.

[9] Alzib AA and Abdelnabi GH (2017). Eimeria Spp. infection in some broiler farms in Khartoum State, Sudan. SOJ Vet Sci; 3 (4): 1-3.

[10] Dalloul RA and Lillehoj HS (2006). Poultry coccidiosis: recent advancements in control measures and vaccine development. Expert Rev. Vaccines; 5 (1): 143-163.

[11] Chapman HD and Jeffers TK (2015). Restoration of sensitivity to salinomycin in Eimeria following 5 flocks of broiler chickens reared in floor-pens using drug programs and vaccination to control coccidiosis. Poult Sci.; 94 (5): 943-946.

[12] Quiroz-Castañeda RE and Dantán-González E (2015). Control of avian coccidiosis: Future and present natural alternatives. Biomed Res Int.; 11 pages, doi: 10.1155/2015/430610.

[13] Chapman HD (2014). Milestones in avian coccidiosis research: a review. Am Hist Rev.; 119 (2): 501-511.

[14] Shirley MW and Harvey DA (1996). Eimeria tenella: infection with a single sporocyst gives a clonal population. Parasitology; 112: 523-528.

[15] NRC (1994). Nutrient Requirements of Poultry. Ninth Revi. Subcommittee on Poultry Nutrition, editor. Washington, DC: Natl. Acad. Press.

[16] Johnson J, Reid WM (1970). Anticoccidial drugs: lesion scoring techniques in battery and floor-pen experiments with chickens. Exp Parasitol.; 28 (1): 30-36.

[17] Peek HW and Landman WJM (2003). Resistance to anticoccidial drugs of Dutch avian Eimeria spp. field isolates originating from 1996, 1999 and 2001. Avian Pathol.; 32 (4): 391-401. 
[18] Ojimelukwe AE, Emedhem DE, Agu GO, Nduka FO and Abah AE (2018). Populations of Eimeria tenella express resistance to commonly used anticoccidial drugs in southern Nigeria. Int J Vet Sci Med.; 0-1.

[19] Ahmed A. Al-Gawad, Olfat A. Mahdy Aida AN El-Massry and Mohamed SA Al-Aziz (2012). Studies on coccidia of egyptian balady breed chickens. Life Sci J.; 9 (3).

[20] Abu-Akkada SS and Awad AM (2012). Isolation, propagation, identification and comparative pathogenicity of five Egyptian field strains of Eimeria tenella from broiler chickens in five different provinces in Egypt. Res Vet Sci.; 92 (1): 92-95.

[21] Bozkurt M, Aysul N, Küçükyilmaz K, Aypak S, Ege G, Çatli AU (2014). Efficacy of in-feed preparations of an anticoccidial, multienzyme, prebiotic, probiotic, and herbal essential oil mixture in healthy and Eimeria spp.-infected broilers. Poult Sci.; 93 (2): 389-399.

[22] Mathis G, Schaeffer J, Cookson K, Dickson J, LaVorgna M and Waldrip D (2014). Effect of lasalocid or salinomycin administration on performance and immunity following coccidia vaccination of commercial broilers. J Appl Poult Res.; 23 (4): 577-585.

[23] Conway DP, Sasai K, Gaafar SM and Smothers CD (1993). Effects of different levels of oocyst inocula of Eimeria acervulina, E. tenella, and E. maxima on plasma constituents, packed cell vo lume, lesion scores, and performance in chickens. Avian Dis.; 37 (1): 118.

[24] Adamu M, Boonkaewwan C, Gongruttananun N and Vongpakorn M (2013). Hematological, biochemical and histopathological changes caused by coccidiosis in chickens. Kasetsart J - Nat Sci.; 47 (2): 238-246.

[25] Hirani ND, Hasnani JJ, Pandya SS and Patel PV (2018). Haematological changes in broiler birds with induced caecal coccidiosis following prophylaxis with different coccidiostats. Int J Curr Microbiol Appl Sci.; 7 (04): 1094-1100.

[26] Mondal DK, Chattopadhyay S, Batabyal S, Bera AK and Bhattacharya D (2011). Plasma biochemical indices at various stages of infection with a field isolate of Eimeria tenella in broiler chicken. Vet World.; 4 (9): 404-409.

[27] Basith AS, Rajavelu G and Manohar MB (1998). Biochemical studies in experimental Eimeria necatrix infection in chickens. Indian Vet J.; 75 (10): 876-878.

[28] Patra G. Rajkhowa, Ayub M, Tiwary JG and Sailo L (2009). Studies on clinical, gross, histopathological and biochemical parameters in broiler birds suffered from Eimeria necatrix infection in Aizawl District of Mizoram, India. Int J Poult Sci.; 8 (11): 1104-6.

[29] Peek HW and Landman WJM (2011). Coccidiosis in poultry: anticoccidial products, vaccines and other prevention strategies. Vet Q.; 31 (3): 143-161.

[30] Györke A, Kalmár Z, Pop LM and Şuteu OL (2016). The economic impact of infection with Eimeria spp. in broiler farms from Romania. Rev Bras Zootec.; 45 (5): 273-280.

[31] Chapman HD Jeffers TK (2014). Vaccination of chickens against coccidiosis ameliorates drug resistance in commercial poultry production. International Journal for Parasitology: Drugs and Drug Resistance; 4 (3): 214-217. 